\title{
Realization of a Hand-held Circular Polarized Antenna's Transmitting Polarization mode Checker
}

\author{
XieYong $^{\text {a }}$, Liu Guoqing ${ }^{b}$, Xue Juncand Ding Ran ${ }^{d}$ \\ Marine Department of Satellite Tracing and Metering, Jiangyin China, 214431 \\ a13915206262@139.com, bLGQ11@163.com, ‘XueJun@163.com, dSYDR@163.com
}

\begin{abstract}
Keywords: Circular Polarization; Polarization mode; Logarithmic Detector; LDO; Shell
Abstract. Circularly polarized antennas are widely used in the field of satellite communications. The receiving and transmitting polarization mode are generally controlled by polarization switch. Compared with the receiving polarization mode detection, the transmitting polarization mode detection is complex relatively and usually requires to establish a test environment in the far field. In this paper, A hand-held polarization mode checker device has been designed to check the transmitting polarization mode in near-field by using the relative difference numerical analysis method, The device includes a collection circuit and a shell. The collection circuit adopts a low-power design and uses the logarithmic demodulation method to complete the analog-to-digital conversion. The shell adopts a dislocation locking method to achieve the screw-free fixation, The polarization mode recognition can be finished by using different means while transmitting and receiving signal. Its greatest advantage lies in its low power consumption, convenient portability, and near-field testability. Test results have shown that the device is simple to use, convenient to test, and reliable in performance, and has great significance to practical test work.
\end{abstract}

\section{Introduction}

Circularly polarized antennas ${ }^{[1]}$ are commonly used in satellite communications for the signal transmission and reception because the linear polarization signal can easily bring waveform distortion when it penetrates the atmosphere. At the same time, in order to meet the needs of different tasks, the polarization switch is used in the RF part to switch between left-handed and right-handed circular polarization $^{[2]}$, and all of these functions are realized by a dedicated controller. But the polarization switch is a non-standard part, it will bring serious consequences for tracking once the left-handed and right-handed circular polarization marks are inconsistent with the actual transmitting polarization mode. In one of the test tasks, the tracking results were abnormal due to the reversed polarization switch control circuit of the antenna, which seriously affected the precision identification work.

This paper proposed a near-field comparison measurement method aiming at the tedious drawbacks of traditional testing methods. A portable antenna transmitting polarization mode detection device has been designed by using a low power circuit board and portable structure.

\section{System Design}

The receiving and transmitting polarization mode detection methods of the circularly polarized antenna are the same. The detection of receiving polarization mode is relatively simple, because the polarization mode of antenna can be distinguished by the left-handed and right-handed antenna's received signal from space targets whose polarization mode and strengths have been known. For the transmitting polarization mode detection, The conventional method usually needs the antenna to transmit power signal, and monitoring the strength of the circular polarized antenna's received signal by a spectrum analyzer in far-field.

When the polarization modes are the same, the signal strength is strong. Because of the complex detection process of the antenna's transmitting polarization mode, a portable device has been designed to complete the detection work in the near field. The principle is shown in Figure 1. The key technology is to detect the signal's power. There are generally two methods for signal strength 
detection: the first detection method is to use the detector tube. This method requires a wide input signal band and has a poor response to small signal, and the processing circuit is also very complex; the second detection method is to use a wide-band logarithmic detection, this method can detect RF signal between $200 \mathrm{MHz}$ to $8 \mathrm{GHz}$. The processing circuit is simple, and can be responded quickly, in addition, there are special chips to support it.

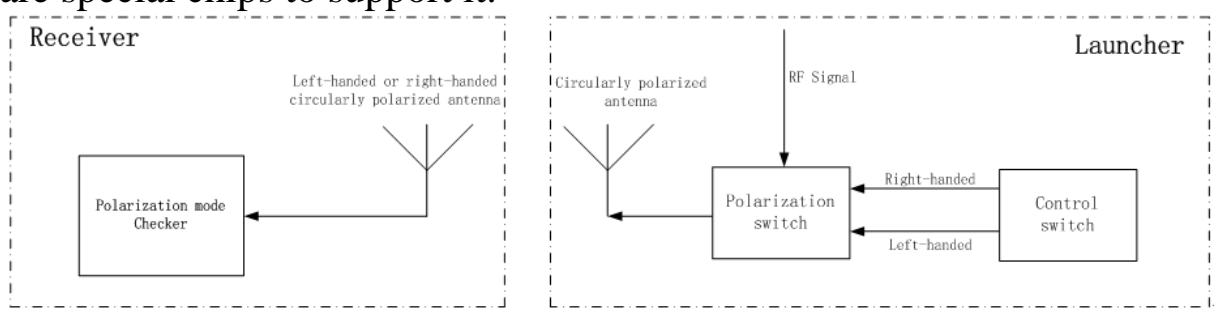

Fig 1 System Block Diagram

According to the practical application scene, a direct logarithmic detection method is adopted to design the circuit board and the shell structure. The main function of the circuit board is to collect the logarithmic detector's output and display it. When using it, the transmitting end controls the polarization switch through the control switch and sends out a signal with one polarization mode, and adjusts the signal power until the receiving end receives the signal. At the receiving end, two different circularly polarized antenna is used, and the antenna's polarization is the same when transmit and different when receive. In this method, the antenna receives a certain polarized signal from the transmitting end; Other method is to use a antenna with a single polarization, whose polarization is same when receiving and different when transmitting, and the polarization can be distinguished by measuring the left-handed and right-handed polarized signal from the transmitting end.

\section{Hardware Circuit Design}

Low Power Circuit Design. The circuit function principle is shown as in Fig2, this control circuit is mainly used to collect the value of the logarithmic detector, control the working state, and display the collected data. According to the requirement of function in the circuit design, the low power design must be considered mainly including the choice of chip, the design of circuit board, the program of control software.

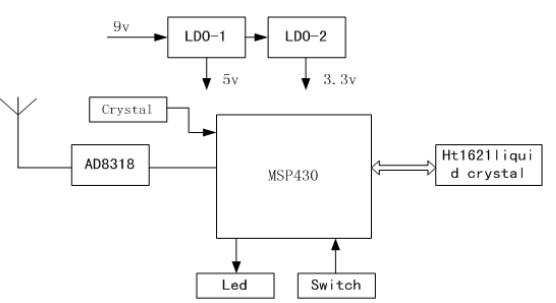

Fig 2 Acquisition circuit functional block diagram

Chip Selection. The MCU selects the MSP430f149 chip to complete the data acquisition of the AD8318's output. The acquired result is displayed on the HT1621, and indicating the current operating status at the same time. In addition to the low-power features such as the sleep function ${ }^{[3][4]}$, it also has a 12-bit ADC function, which can provide high detection data accuracy.

The logarithmic detector uses the AD8318 to monitor the RF signal power and convert it to a DC voltage between $0.2 \mathrm{~V}$ and $2 \mathrm{~V}$ at the output. The frequency range is $1 \mathrm{MHz}$ to $8 \mathrm{GHz}$; the dynamic range is greater than $55 \mathrm{~dB}$; the impulse response's rising time is $12 \mathrm{~ns}$ and the fall time is $10 \mathrm{~ns}$; the operating temperature is $-40^{\circ} \mathrm{C}$ to $+85^{\circ} \mathrm{C}$; the temperature stability is $\pm 0.5 \mathrm{~dB}$; the power consumption is $68 \mathrm{~mA} @+5 \mathrm{~V}$.

The liquid crystal display module selects the HT1621, which is a 128-point memory-mapped and versatile LCD driver. The software configuration features of the HT1621 make it suitable for a variety of LCD applications, including LCD modules and display subsystems. There are only 4 or 5 pins to connect the host controller and the HT1621. The HT1621 also has a power-saving command to reduce system power consumption. 
According to the system power supply's requirements, which are $5 \mathrm{~V}$ and $3.3 \mathrm{~V}$, a linear voltage-stabilized method is adopted. Through the selection of differential voltage, noise, quiescent current, and CMRR, especially quiescent current. the supplying of 5V adopts the HT7150 regulator chip and the supplying of 3.3V adopts TPS79733 regulator chip, the principle shown in Fig 3.
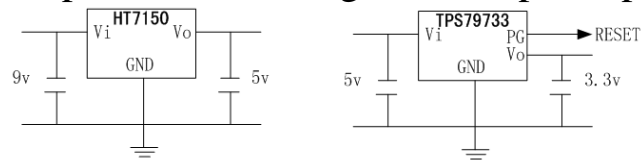

Fig 3 Power Supply Block Diagram

Board layout. The wiring uses digital circuits and microwave circuits to simulate isolation between ground and digital ground, in order to avoid the digital circuits' influence on microwave circuits.

Software program control. In order to ensure the minimum power consumption of the device when it is idle, it is necessary to distinguish between the working state and the hibernation state during program design. In the hibernation state, it is necessary to ensure there is no power consumption.

Software Processing Procedure. The programming of the single-chip microcomputer(SCM) should make the whole circuit work stably and orderly, so it is necessary to consider the working principle and the whole time-sequence requirement of each circuit unit. The working principle of the device is shown as follows: The RF antenna receives the RF signal, the signal is converted by the AD8318, and the converted data is sent to the MCU for ADC conversion. The transformed data is visually displayed through the screen. It is thus clear that the MCU is in charge of the ADC conversion and driving the LCD to display data. The procedure is shown in Fig 4 .

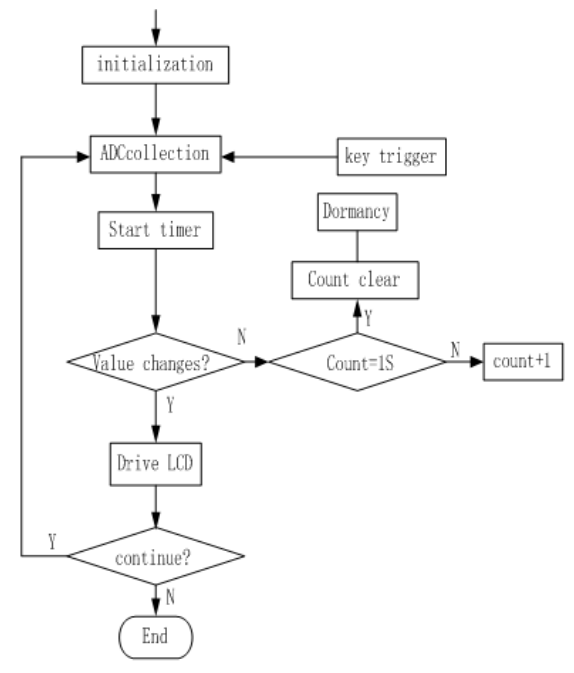

Fig 4 Software process

The most pivotal point of the program is using the output value of the AD8318 to carry out ADC conversion through the single channel in real time. The principle is shown in Fig 5 and the referenced voltage source is $5 \mathrm{~V}$. Due to the use of relative numerical comparisons, the AD8318's conversion ratio and calibration are not necessary to be considered during program design. For the single-channel ADC conversion of the external referenced source, in ADC12, $\mathrm{Vr}+=\mathrm{VeREF}+, \mathrm{Vr}-=\mathrm{Avss} ; \mathrm{Vr}+$ and Vr- are the maximum and minimum referenced voltage source values of the ADC12 module. When the analog voltage of the input signal is equal to or higher than $\mathrm{Vr}+$, the ADC's output is converted to full-scale output and the output value is 0x0FFF. When the analog voltage of the input signal is equal to or lower than Vr-, the ADC's output value is 0, and the output value is 0x0000. The ADC12's analog voltage conversion formula is: $\mathrm{Nadc}=4095 \times(\mathrm{Vin}-\mathrm{Vr}-),(\mathrm{Vr}+-\mathrm{Vr}-)^{[5]}{ }^{[6]}$.

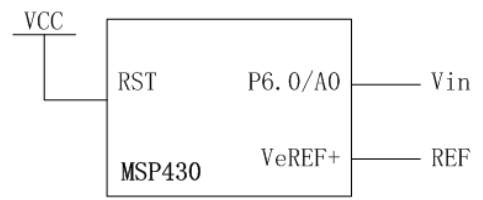

Fig 5 AD Acquisition Block Diagram 


\section{Structural Design}

The shell includes a front cover and a rear cover, both of them use a square structure, as shown in figure 6 . The front cover is composed of a shell, a salient bar, a lock bar, a display window, and a SMA connector. The salient bar has a mouse-shaped structure at the bottom in order to facilitate the locking and the locking of the sinker with the rear cover; the locking bar is in the shape of "L". The shape can not only facilitate plugging and pulling out the collection circuit board and the display circuit board, but also block the movement of them. The display window has a rectangular frame on the outer casing, and the edge of the rectangular frame has a "Z-shaped" structure. The rear cover includes a shell and a sink. The sink is a semi-closed structure. The sink is shaped like a mouse in the top view and has a locking strip in the center of the closed space, it is convenient to lock with the ribs of the front cover.

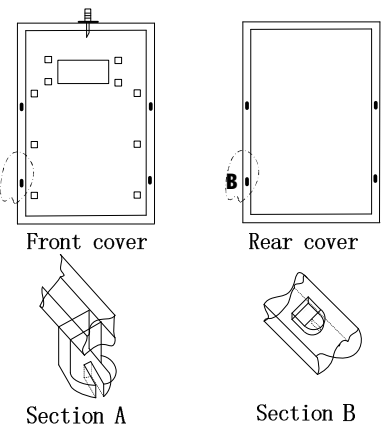

Fig 6 Shell Structure

\section{Test verification}

The test environment is composed of a signal source, an antenna for transmitting, two antennas for receiving (the antenna gain is basically the same, the attenuation of the feeder is basically the same too), and a spectrum analyzer and a handheld device.

The signal source is set to a radio frequency, the transmitting antenna is right-handed circularly polarized, and the receiving antenna is right-handed circularly polarized and left-handed circularly polarized. The test results show that the right-handed detection voltage is $1.23 \mathrm{~V}$, and the intensity is about $-27 \mathrm{dBm}$; the left-handed detection voltage is $1.88 \mathrm{~V}$, and the intensity is approximately $-54 \mathrm{dBm}$, all indicate that the transmitting polarity is right-handed.
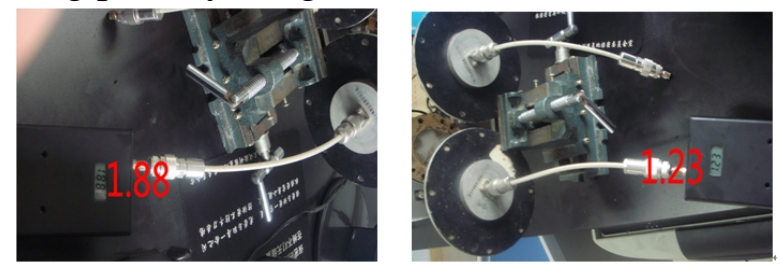

Fig 7 Left-handed, right-handed receiving antenna test results

Comparing with the test results of the spectrum analyzer (as shown in fig8), the spectrum analyzer tests results show that the right-handed data is $-24.4 \mathrm{dBm}$ and left-handed data is $-35.9 \mathrm{dBm}$, which also indicate that the transmitting polarity is right-handed.

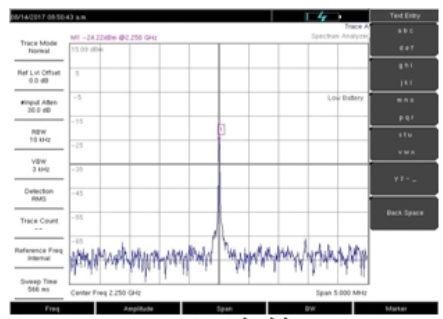

(1) 右旋

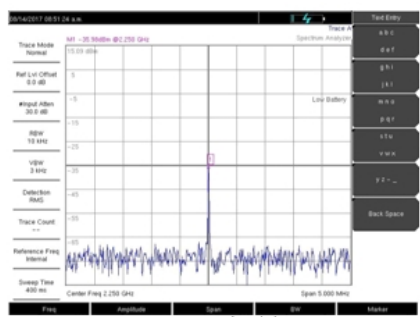

(2) 左旋

Fig 8 Right-handed, left-handed spectrometer measurement results 


\section{Conclusions}

This paper introduces a design method of a hand-held circular polarized antenna's polarization mode inspection device. The device is composed of a shell and an acquisition board. A lock bar structure design is adopted, and the polarization mode can be distinguished from the received power difference between both polarization. Place the two flanking of the display board on the lower part of the front cover's lock bar. Push the display circuit board up to lock the flanking into the lock bar. Put the two flanking of the acquisition circuit board on the upper part of the front cover's lock bar. Push the display circuit board down to lock the flanking into the lock bar. Connect the acquisition circuit board and the display circuit board with a row cable, A SMB connector is used for the RF output of the collection circuit board, and it is a SMA connector to the outside. Put the locking bar of the rear cover into the ribs of the front cover and push the rear cover down to form an integral device. The greatest advantage of this device is that it can receive the left-handed or right-handed signal from the antenna under test through a circularly antenna whose polarization has been known, and to use the difference in the received signal strength to know whether the polarization switch's control is correct. It can be installed without tools and has a great place in works such as replacing the battery.

\section{Acknowledgements}

This work was financially supported by the Marine Department of Satellite Tracing and Metering.

\section{References}

[1] Haupt R L.An Introduction to Genetic Algorithms for Electromagnetics[J].IEEE Trans on Antennas and Propagation,1995,37(2):7-15.

[2] Haupt RL.Thinned Arrays Using Genetic Algorithms[J].IEEE Trans on Anennas and Propagation, 1994,42(7):993-999.

[3] MSP430x4xx Family User's Guide (SLAU056B).

[4] MSP430x44x datasheets (SLAS344). 2002. 1.

[5] Wei Xiaolong. Design example of MSP430 series microcontroller interface and system[M]. Beijing University of Aeronautics and Astronautics Press, 2002. 11.

[6] Tan Haoqiang. C Programming Design (2nd Edition) [M]. Tsinghua University Press, 1999, 12. 